\title{
Influencia de la identidad grupal en la cohesión: estudio piloto
}

\section{Influence of Group Identity in Cohesion: Preliminary Study}

\author{
M. Rocío Bohórquez, Macarena Lorenzo, M. Reyes Bueno y Miguel Á. Garrido.
}

Universidad de Sevilla, España

\begin{abstract}
Resumen: La cohesión grupal es uno de los procesos dinámicos más estudiados en los equipos deportivos, su relación con procesos como el clima grupal o la eficacia colectiva ha sido ampliamente abordada. Sin embargo, el estudio de la influencia que la identidad grupal pudiera tener sobre la cohesión está aún por emprender. Así, el objetivo de este estudio fue determinar la influencia que la identidad grupal pudiera tener sobre la cohesión de los equipos deportivos. En el experimento participaron 51 estudiantes de primer curso del Grado de Ciencias de la Actividad Física y el Deporte como parte de las prácticas obligatorias de la asignatura Psicología Social del Deporte; los participantes fueron divididos en cuatro equipos distribuidos aleatoriamente que competían dos a dos; dos equipos crearon libremente su identidad grupal mientras que a los otros dos se les impuso. Los resultados muestran la influencia significativa que la imposición o no de una identidad grupal en los equipos deportivos tiene sobre la cohesión en sus diferentes dimensiones $(Z=-2,768, p<0,01$ para la atracción individual hacia la tarea; $Z=-3,649, \mathrm{p}<0,01$ para la atracción individual hacia lo social, $Z=-2,570, p<0,01$ para la atracción grupal a la tarea y $Z=-1,981$, $\mathrm{p}<0,05$ para la atracción grupal hacia lo social). Así, la identidad grupal se muestra como una variable manipulable a tener en cuenta en el trabajo de teambuilding.

Palabras clave: identidad grupal, cohesión, equipos deportivos.
\end{abstract}

Abstract: Group cohesion is one of the most studied dynamic processes in sports teams, its relationship with processes such as motivational climate or collective efficacy has been widely addressed. However, the study of the influence of static group elements have on cohesion has yet to address, so it would be necessary to study the relationship between cohesion and static elements such as group norms, or differentiating reference elements, defining group identity of a team. The aim of this study was to determine the influence of group identity may have on the cohesion of sports teams. Participants were 51 pupils of Science Degree in Physical Activity and Sport as part of the practices of the subject Social Psychology of Sport; they werewere divided randomly into four teams that competed two to two. Two teams developed their group identity freely while the other two were imposed. The results show the significant influence that the imposition or not of a group identity in sports teams have on cohesion in its different dimensions $(\mathrm{Z}=-2.768, \mathrm{p}<0.01$ for individual attraction to the task, $\mathrm{Z}=-3.649, \mathrm{p}$ $<0.01$ for individual attraction to the social, $Z=-2.570, p<0.01$ for the attraction to the task group and $Z=-1.981, p<0.05$ for the attraction to the social group). Thus, group identity is shown as a manipulated variable to consider in the work of team building.

Key words: group identity, cohesion.

\section{Introducción}

La identidad social fue definida por Tajfel (1981) como el conocimiento que se tiene de pertenecer a un determinado grupo social. Posteriormente, Turner (1982) postuló que esta identidad social era de ser necesariamente entendida de forma diferente cuando se refería a relaciones grupales interactivas producidas dentro del propio grupo y no en su relación con otros grupos o el contexto social, en este caso estaríamos hablando de identidad grupal.

La identidad grupal está determinada por la estructura grupal (Fields, 2011): tamaño del grupo, homogeneidad/heterogeneidad de sus miembros, normas grupales, distintividad, etc.; elementos todos ellos que han sido estudiados en el contexto deportivo por su relación con elementos grupales dinámicos como la cohesión (Carron, Shapcott y Burke, 2007) o la eficacia colectiva (Beauchamp, 2007). La configuración de estos elementos estáticos en una identidad grupal determinada tendrá influencia sobre la cohesión grupal: no es posible

Contacto: M. Rocío Bohórquez Gómez-Millán. Departamento de Psicología Social, Facultad de Psicología, Universidad de Sevilla. C/Camilo José Cela S/N, 41018, Sevilla. E-mail: rociobohorquez@us.es. Tlf.: +34 954 557761. estar cohesionado en un grupo si no se pertenece a él al nivel psicológico.

La cohesión es un proceso dinámico que se refleja en la tendencia de un grupo a mantenerse unido en la consecución de sus objetivos instrumentales y/o para la satisfacción de las necesidades afectivas de sus miembros (Carron et al, 2007). Cada miembro del equipo desarrolla una percepción sobre el equipo que está relacionada con el equipo como totalidad y la manera en que la asociación cumple las necesidades y objetivos personales; estas percepciones se configuran en cuatro categorías: integración grupal (percepción individual acerca de la cercanía, la similitud y la unión dentro del grupo como un todo), la atracción individual hacia el grupo (percepción individual sobre las motivaciones y sentimientos personales que atraen al individuo hacia el grupo), orientación a la tarea (orientación general hacia el logro de los objetivos del grupo) y orientación hacia lo social (orientación general hacia el desarrollo y mantenimiento de relaciones y actividades sociales dentro del grupo), (Carron et al. 2007, Carron et al. 2007b). Estas percepciones conforman los factores atracción individual a la tarea, atracción individual a lo social, atracción grupal a la tarea y atracción grupal a lo social (Carron, Widmeyer 
y Brawley, 1985).

Si finalmente la identidad grupal tiene influencia sobre los procesos de cohesión en los equipos deportivos, este aspecto como estructura previa debe ser abordado en los programas de team building que ya contemplan aspectos como el manejo de normas, el establecimiento de objetivos comunes o la integración grupal (Estabrooks, 2007).

El objetivo del estudio fue, por tanto, explorar la influencia que la identidad grupal pudiera tener sobre la cohesión de los equipos deportivos.

\section{Método}

\section{Participantes}

En el estudio participaron 57 estudiantes de primer curso de Grado en Ciencias de la Actividad Física y el Deporte, concretamente alumnos de la asignatura Psicología Social del Deporte en el contexto de las clases prácticas de la asignatura. De estos estudiantes, 45 eran hombres y 5 mujeres, con edades comprendidas entre 18 y 47 años $(M=19.46$, DT $=4.12)$.

\section{Instrumentos}

El instrumento empleado para este estudio fue el Group Environment Questionnaire (GEQ) (Carron, Widmeyer yBrawley, 1985). El GEQ evalúa cuatro elementos en relación con lo atractivo que es un grupo de sus miembros. Mide la atracción del individuo hacia el grupo, así como la integración del grupo en lo relativo a la tarea y la dimensión social en cuatro subescalas diferentes: la atracción individual hacia el trabajo, la atracción individual hacia lo social, la integración del grupo en la tarea y la integración del grupo a nivel social.

La monitorización de los elementos propios de la identidad grupal como normas, existencia y utilización de símbolos característicos y existencia y utilización de espacios propios, se llevó a cabo mediante registro de frecuencias en una tabla de registro diseñada ad hoc. Se tuvieron en cuenta el seguimiento de normas grupales (internas de funcionamiento, relativas a la uniformidad y normas relacionadas con el seguimiento de reglas de juego) y la utilización de espacios propios de cada equipo -foro privado- dentro de la sección de la asignatura en la plataforma de enseñanza virtual habilitada por la Universidad.

\section{Procedimiento}

De forma previa al inicio de las prácticas, los participantes fueron divididos aleatoriamente en 4 equipos que se enfrentarían 1 a 1 (una dupla en cada turno de clase) durante las 15 sesiones previstas para el desarrollo de éstas, diseńadas en formato de competición liguera multideporte. Cada sesión práctica se proponía un encuentro deportivo en el que se combinaban objetivos de trabajo técnico-táctico con objetivos de aprendizaje relativos a los contenidos de la asignatura, la victoria en el encuentro sumaba tres puntos al cómputo del equipo, el empate un punto y la derrota ninguno.

En la primera sesión de prácticas, se indicaron tareas diferentes para cada equipo:

a) Un equipo de cada turno de prácticas debía diseñar sus propios elementos de identidad grupal: nombre, colores, himno, escudo, normas, etc. Equipos 2 del primer turno y 4 del segundo turno.

b) El otro equipo de cada turno debía estudiar los elementos de identidad de su equipo, elementos impuestos por el equipo docente de la asignatura. Equipos 1 del primer turno y 3 del segundo turno.

Durante las siguientes prácticas, no se hizo referencia explícita alguna a las identidades de cada equipo mas allá de dirigirse a ellos por el nombre que habían elegido o se les había impuesto.

Para explorar la influencia de la identidad grupal sobre la cohesión de estos equipos, se utilizó un diseño pretest-postest, realizándose el pretest en la tercera sesión práctica y el postest en la decimoquinta (y última). Además, con el objetivo de monitorizar los indicadores de identidad grupal de los equipos, se llevó a cabo un seguimiento del cumplimiento de las normas grupales y de la utilización de espacios propios de cada equipo.

De forma previa al análisis de los datos se realizaron pruebas de normalidad y homocedasticidad de la muestra con el fin de verificar la posibilidad de usar pruebas paramétricas. Se determinó una distribución no normal de la muestra, así como la no homogeneidad de varianzas (pruebas de Kolmogorov-Smirnov y Levene, $\mathrm{p}<0.05$ ); por ello se decidió utilizar pruebas no paramétricas en los análisis estadísticos realizados para este estudio. El análisis de los datos se llevó a cabo con el paquete estadístico de análisis SPSS 17.0 (StatisticalPackagefor Social Sciences).

\section{Resultados}

Con el fin de conocer la influencia que la identidad grupal pudiera tener sobre la cohesión, se compararon los datos obtenidos en el cuestionario GEQ por los cuatro equipos participantes. Los resultados señalan diferencias significativas entre estos equipos en los factores Atracción individual a lo social y Atracción grupal a la tarea (ver tabla 1).

Tabla 1. Comparación de factores de cohesión grupal entre equipos.

\begin{tabular}{lcl}
\hline & $\mathrm{X}^{2}$ & Sig. \\
\hline Atracción individual a la tarea & 7.602 & .055 \\
Atracción individual a lo social & 18.357 & $.000^{*}$ \\
Atracción grupal a la tarea & 7.866 & $.049^{* *}$
\end{tabular}




\begin{tabular}{lcc}
\hline & $\mathrm{X}^{2}$ & Sig. \\
\hline Atracción grupal a lo social & 6.510 & .089 \\
${ }^{*} \mathrm{p}<.01^{* *} \mathrm{p}<.05$ & &
\end{tabular}

Para determinar si dichos cambios tenían relación con el desarrollo diferencial de la identidad grupal por parte de los diferentes equipos, se realizaron comparaciones entre aquellos enfrentados en cada turno de prácticas (recuérdese, por cada turno un equipo con identidad impuesta y otro equipo con elementos de identificación elegidos libremente). Los resultados muestran diferencias significativas en los factores Atracción individual a la tarea, Atracción individual a lo social y Atracción grupal a la tarea entre los equipos del primer turno de prácticas, por el contrario, no aparecen diferencias significativas entre los equipos correspondientes al segundo turno de prácticas (ver tabla 2).

Tabla 2. Comparación de factores de cohesión grupal entre equipos con identidad impuesta y equipos con elementos de identidad libremente escogidos.

\begin{tabular}{llcc}
\hline & & $\mathrm{Z}$ & Sig. \\
\hline \multirow{2}{*}{$\begin{array}{l}\text { Equipos } \\
\text { rivales }\end{array}$} & Atracción individual a la tarea & -2.768 & $.006^{*}$ \\
\cline { 2 - 4 } $1^{\circ}$ turno & Atracción individual a lo social & -3.649 & $.000^{*}$ \\
& Atracción grupal a la tarea & -2.570 & $.010^{* *}$ \\
& Atracción grupal a lo social & -1.981 & .048 \\
\hline \multirow{2}{*}{$\begin{array}{l}\text { Equipos } \\
\text { rivales }\end{array}$} & Atracción individual a la tarea & -.275 & .783 \\
$2^{\circ}$ turno & Atracción individual a lo social & .607 & .544 \\
& Atracción grupal a la tarea & -.000 & 1.000 \\
\cline { 2 - 2 }${ }^{*} \mathrm{p}<.011^{* *} \mathrm{p}<.05$ & -.138 & .890 \\
\hline
\end{tabular}

La monitorización de elementos propios de la identidad grupal de los equipos revela adhesión desigual a éstos: mientras que los equipos del primer turno tienden al seguimiento de normas grupales y al uso de los espacios privados de grupo, los equipos del segundo turno parecen no hacer un uso tan habitual de los espacios propios (ver figuras 1 y 2).

Figura 1. Seguimiento de normas por parte de los equipos.

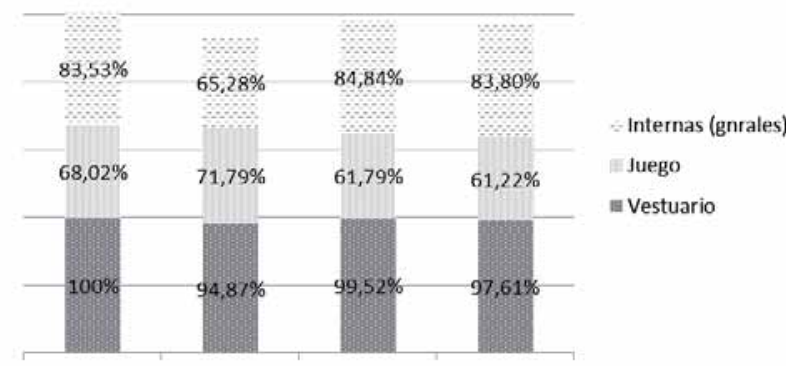

Eq 1. Turno 1 Eq.2. Turno 1 Eq.3. Turno 2 Eq.4. Turno 2
Figura 2. Uso de espacios propios de los equipos: foro de los equipos en el espacio web de la asignatura.

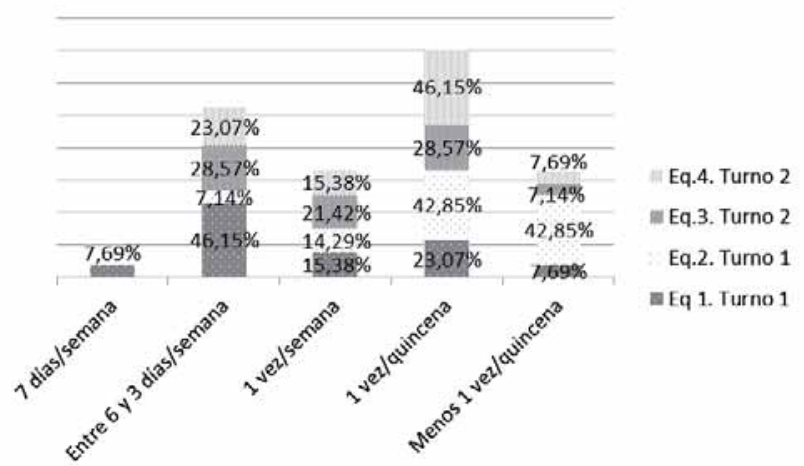

\section{Discusión}

Este trabajo tenía como objetivo explorar la influencia que la identidad grupal de un equipo pudiera tener sobre su cohesión grupal. Los resultados indican que los equipos sometidos a procesos diferenciales de construcción de identidad desarrollan distintos niveles de cohesión que aquellos cuya identidad grupal les ha sido impuesta. Estas diferencias se expresan en las dimensiones referidas a las percepciones que tienen sus miembros acerca de motivaciones comunes que les llevan a sentirse atraídos por la pertenencia a su grupo y las emociones personales relativas a esa pertenencia (atracción individual hacia lo social) así como en la orientación general del equipo hacia el objetivo y la tarea que éste desarrolla (atracción grupal a la tarea).

El análisis de las dimensiones de cohesión entre equipos con identidad impuesta frente a aquellos que la eligieron libremente muestra que existen diferencias significativas en el caso del $1^{\circ}$ turno de prácticas, pero no en el $2^{\circ}$ turno. Estas diferencias pueden ser explicadas a la luz de la información aportada por el seguimiento de los elementos de identidad grupal: mientras que los dos equipos del $1^{\circ}$ turno de prácticas muestra seguimiento de normas grupales y utilización frecuente de los espacios privados, ambos equipos del $2^{\circ}$ turno usan el foro privado de manera puntual. Aunque no se pueda afirmar con rotundidad, esta diferencia en cuanto a elementos componentes de la identidad grupal parece indicar que los equipos del primer turno de prácticas desarrollaron una identidad grupal más fuerte, y esas identidades contrapuestas serían la causa del desarrollo diferencial de su cohesión grupal. Así, sólo cuando la identidad asumida (sea impuesta o elegida) se haya desarrollado, se podrán encontrar diferencias en lo que a cohesión social se refiere.

Este estudio preliminar muestra ciertas debilidades que deberían ser subsanadas en un estudio en profundidad como el reducido tamaño de la muestra o la carencia de instrumen- 
tos de evaluación de los elementos estáticos de los grupos, concretamente de la identidad grupal que en esta ocasión ha sido inferida por la presencia/ausencia de sus elementos definitorios. A pesar de ello, este trabajo aporta una visión clara acerca de la importancia de tener en cuenta la identidad grupal como variable determinante de la cohesión del equipo que debería ser tenida en cuenta en el diseño y desarrollo de programas de teambuilding.

\section{Referencias}

Beauchamp, M. R. (2007). Efficacy beliefs within relational and group contexts in sport. En S. Jowett y D. Lavallee (Ed.). Social Psychology in Sport. Champaign, IL.: Human Kinectics.

Carron, A. V.; Shapcott, K. M.; Burke, S. M. (2007). Group cohesion in sport and exercise: past, present and future.En M. R. Beauchamp y M. A. Eys (Ed.).Group Dynamics in exercise and sport psychology. New York: Routledge.

Carron, A.V., Widmeyer, W.N., \& Brawley, L.R. (1985). The development of an instrumentto assess cohesion in sport teams: The group environment questionnaire. Journal of Sport Psychology, 7, 244-266.

Estabrooks, P. A. (2007). Group integration interventions in exercise: theory, practice and future directions. En M. R. Beauchamp y M. A. Eys (Ed.).Group Dynamics in exercise and sport psychology.New York: Routledge.

Fields, D. A. (2011).Trajectories of identification across social spaces: Intersections between home, school and everyday spaces. Dissertation Abstracts International Section A: Humanities and Social Sciences, 72 (4A).

Tajfel, H. (1981). Human groups and social categories. Cambridge: Cambridge University Press.

Turner, J. C. (1982). Towards a cognitive redefinition of the social group. En H. Tajfel (Ed.). Social identity and intergroup relations.Cambridge: Cambridge University Press. 